\title{
Protective immunity against Toxoplasma gondii induced by DNA immunization with the gene encoding a novel vaccine candidate: calcium- dependent protein kinase 3
}

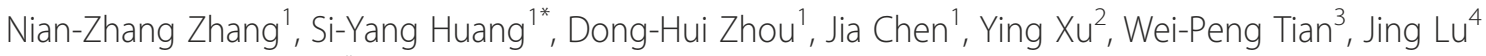 \\ and Xing-Quan Zhu $u^{1,2,5^{*}}$
}

\begin{abstract}
Background: Toxoplasma gondii can infect almost all warm-blood animals including human beings. The plant-like calcium-dependent protein kinases (CDPKs) harbored by T. gondii are involved in gliding motility, cell invasion, egress and some other developmental processes, and so have been implicated as important virulence factors.

Methods: In the present study, we constructed a DNA vaccine expressing T. gondii CDPK3 (TgCDPK3) and evaluated its protective efficacy against T. gondii infection in Kunming mice. The gene sequence encoding TgCDPK3 was inserted into the eukaryotic expression vector pVAX I, and mice were immunized with pVAX-CDPK3 intramuscularly.

Results: The results showed that mice immunized with pVAX-CDPK3 developed a high level of specific antibodies and a strong lymphoproliferative response. The significantly increased levels of IFN- $\gamma, \mathrm{IL}-2, \mathrm{LL}-12$ (p70) and IL-23 and high ratio of IgG2a to lgG1 antibody titers indicated that a Th1 type response was elicited after immunization with pVAX-CDPK3.

Furthermore, the percentage of CD4+ T cells in mice vaccinated with pVAX-CDPK3 was significantly increased. After lethal challenge with the tachyzoites of the virulent $T$. gondii RH strain, the mice immunized with pVAX-CDPK3 prolonged the survival time from 10 days to 24 days $(13.5 \pm 4.89)$ compared to untreated mice or those received PBS or PVAX I which died within 7 days $(P<0.05)$. In chronic infection model (10 cysts of the T. gondii PRU strain), the numbers of brain cysts of the mice immunized with PVAX-CDPK3 reduced significantly when compared with those in control groups $(P<0.05)$, and the rate of reduction could reach to about $50 \%$.
\end{abstract}

Conclusions: TgCDPK3 can generate protective immunity against acute and chronic T. gondii infection in Kunming mice and is a promising vaccine candidate for further development of an effective vaccine against $T$. gondii.

Keywords: Toxoplasma gondii, Toxoplasmosis, TgCDPK3, DNA vaccine, Protective immunity

\section{Background}

Toxoplasma gondii, an obligate intracellular protozoan parasite, is responsible for toxoplasmosis in a wide range of hosts including humans, mammals, birds and marine mammals [1-5]. T. gondii infection in immune-competent

\footnotetext{
* Correspondence: huangsiyang@caas.cn; xingquanzhu1@hotmail.com 'State Key Laboratory of Veterinary Etiological Biology, Key Laboratory of Veterinary Parasitology of Gansu Province, Lanzhou Veterinary Research Institute, Chinese Academy of Agricultural Sciences, Lanzhou, Gansu Province 730046, PR China

${ }^{2}$ College of Animal Science and Technology, Anhui Agricultural University, Hefei, Anhui Province 230036, PR China

Full list of author information is available at the end of the article
}

individuals is rarely symptomatic, but toxoplasmosis occurred in fetus and immunocompromised hosts may result in severe disease or even lethal damage [5-7]. Meanwhile, the infection can cause serious economic losses to the livestock industry, especially in sheep and goats, as the course of abortion, stillbirth and neonatal loss [8], and also the infected animals are major sources of $T$. gondii transmission to humans $[4,6]$.

No available chemical treatments could completely eliminate $T$. gondii in infected animals, so immunoprophylaxis is considered to be high priority for prevention and control of the parasite $[9,10]$. Although the only licensed vaccine

\section{Ciomed Central}


based on the attenuated-live T. gondii S48 strain (Toxovax ${ }^{\circ}$ ) can be used to prevent the incidence of abortion in sheep [11], it is limited to be further explored in food-producing animals or humans in view of the safety concerns on its reverting to a virulence wild type. The current efforts have been made on the development of DNA vaccines due to the superiority of much safer than live-type vaccines, as well as their ability to induce primarily Th1 cell-mediated immune and CD8+ cytotoxic $\mathrm{T}$ cells (CTL) responses [12,13].

A family of calcium-dependent protein kinases (CDPK) is known as key effectors in regulating calcium related signaling pathways in apicomplexan, which control a diverse array of functions in the life cycle including gliding motility, cell invasion, egress and some other developmental processes that occur at distinct stages in their complex life [14]. $\operatorname{TgCDPK} 3$, a characteristic member of CDPKs, is localized to the parasite periphery in intracellular and extracellular parasites and partially to the apical end of the intracellular parasite [15]. The TgCDPK3 knockout strain showed fewer parasites per vacuole than parental strains, which implied that the gene can partially influence the division of T. gondii [15]. However, no studies have evaluated the immunogenicity of TgCDPK 3 and its potential as a vaccine candidate against $T$. gondii infection.

In this context, the objectives of the present study were to examine the various immune responses in mice induced by DNA immunization with a eukaryotic plasmid expressing TgCDPK3, and to evaluate the potential of $\operatorname{TgCDPK} 3$ as a vaccine candidate against infection with two different genotypes of $T$. gondii in Kunming mice.

\section{Methods}

\section{Animals}

Specific-pathogen-free (SPF) female inbred Kunming mice of 6-8 weeks old were purchased from Center of Laboratory Animals, Lanzhou Institute of Biological Products, Lanzhou, China. All mice were handled in strict accordance with the Good Animal Practice requirements of the Animal Ethics Procedures and Guidelines of the People's Republic of China. This study was approved by the Animal Ethics Committee of Lanzhou Veterinary Research Institute, Chinese Academy of Agricultural Sciences (Approval No. LVRIAEC2012-011).

\section{Parasites}

T. gondii RH and PRU strains were used in this study. Tachyzoites of the highly virulent $T$. gondii $\mathrm{RH}$ strain (Type I) were propagated by serial intraperitoneal passage in Kunming mice. The peritoneal fluid of mice was centrifuged for $10 \mathrm{~min}$ at $1000 \times g$ at $4^{\circ} \mathrm{C}$ to remove the cellular debris and then re-suspended in sterile phosphatebuffered saline (PBS). The obtained tachyzoites were also used for Toxoplasma lysate antigen (TLA) preparation according to our previous studies [16] and total RNA extraction was followed by the instruction of the RNAprep Pure Tissue Kit (TIANGEN, China) manual. Cysts of the PRU strain (Type II) were maintained in the laboratory by oral passage of infective brain homogenate in Kunming mice.

\section{Construction of the eukaryotic expression plasmid}

The complete open reading frame (ORF) of TgCDPK3 was amplified by reverse transcription-polymerase chain reaction (RT-PCR) using primers K3F (5'-GCGGGTAC CATGGCGGATCCGCTCTCGTTCTTCAAC-3') and K3R (5'-GGGCGGCCGCTCACTCATGTTGCGACTCAC-3'), in which the Kpn I and Not I restriction sites were introduced and shown in italic, respectively. After purification using the TIANquick Midi Purification Kit (TIANGEN, China), the PCR products were inserted into pVAX I (Invitrogen) through Kpn I and Not I sites. The resulting plasmid was named pVAX-CDPK3. The concentration of extracted pVAX-CDPK3 was determined by spectrophotometry at OD260 and OD280. The plasmids were diluted with sterile phosphate buffered saline (PBS) to a final concentration of $1 \mu \mathrm{g} / \mu \mathrm{l}$ and stored at $-20^{\circ} \mathrm{C}$.

\section{Expression of pVAX-CDPK3 in vitro}

Recombinant plasmid pVAX-CDPK3 was transfected into HEK293 cells grown in 6-well plates using lipofectamine 2000 reagent (Invitrogen) according to the manufacturer's instructions. The expression of pVAX-CDPK3 in vitro was assayed by indirect immunofluorescence assay (IFA) at 48 after transfection. The protocol of IFA followed previous report [16]. Briefly, the transfected cells were firstly fixed with $100 \%$ acetone and washed with PBS-0.1\% Triton-X-100 (PBST). Then goat anti-T. gondii tachyzoites polyclonal antibody (1: 50 dilution in PBST) and fluorescein isothiocyanate (FITC) labeled rabbit anti-goat mouse IgG antibody (1:2 000, Sigma, USA) were added into each well in proper order. As a negative control, the HEK293 cells were transfected with PVAX I.

\section{Immunization and challenge}

A total of 100 female Kunming mice were randomly divided into four groups ( 25 per group). For the experimental group, mice were immunized with $100 \mu \mathrm{l}$ $(100 \mu \mathrm{g})$ pVAX-CDPK3 by intramuscular injections and boosted with a two-week interval. Mice were injected with empty pVAX I vector or PBS as negative control groups, and the blank control group received nothing. The sera of all groups were collected from the mouse tail vein prior to each immunization and stored at $-20^{\circ} \mathrm{C}$ for ELISA. Pre-immune serum samples were used as negative controls. 
Two weeks after the third inoculation, ten mice in all groups were challenged intraperitoneally with $1 \times 10^{3}$ tachyzoites of the virulent $T$. gondii $\mathrm{RH}$ strain and the survival time were recorded daily until all mice were dead. Three mice of each group were inoculated orally with 10 tissue cysts of the PRU strain and the brain cysts were determined at four weeks after the challenge.

\section{Expression and purification of TgCDPK3 protein in bacteria}

The obtained whole coding region was inserted into the prokaryotic expression vector pET-30a, formed pETCDPK3, and then transformed into E. coli BL21 (DE3) strain and grown in Luria Bertani (LB) with $25 \mu \mathrm{g} / \mathrm{ml}$ kanamycin (Sangon, China). The expression of recombinant $\operatorname{TgCDPK} 3$ (rTgCDPK3) was under the condition of $0.6 \mathrm{mM}$ IPTG (Sangon, China) and shaking for 8 hour at $35^{\circ} \mathrm{C}$. The $\mathrm{rTgCDPK} 3$ protein was purified using NiNTA His bind resin (Novagen) according to the manufacturer's instructions. The products were visualized by the sodium dodecyl sulfate-polyacrylamide gel electrophoresis (SDS-PAGE).

\section{Immunoblotting analysis of the recombinant protein}

The reactogenicity of $\mathrm{rTgCDPK} 3$ protein was then detected by immunoblotting. Following SDS-PAGE, rTgCDPK3 protein was electrotransferred onto nitrocellulose (NC) membranes (Pall, USA). Nonspecific binding sites were blocked with $5 \%$ bovine serum albumin (BSA) in PBS for $1 \mathrm{~h}$ at room temperature (RT). The NC membranes were then incubated with the sera of immunized mice on week 6 (diluted in 1:1 000) for $1 \mathrm{~h}$ at RT. After being washed 3 times with PBST (0.5\% Tween 20 in $\mathrm{PBS})$, the membranes were incubated with diluted secondary antibody (goat anti-mouse IgG-HRP, Sigma, USA; $1: 5000$ ) for $1 \mathrm{~h}$ at RT. Proteins were visualized with 4-chloro-1-naphthol (4-CN, Sangon, China) according to the manufacture' instruction. Sera collected before vaccinations were used as negative control.

\section{Antibody assays}

rTgCDPK3-specific humoral immune response was evaluated by ELISA using SBA Clonotyping System-HRP Kit (Southern Biotech CO., LTD, Birmingham, USA) according to the manufacture's instruction. A total of $100 \mu \mathrm{l}$ rTgCDPK3 $(10 \mu \mathrm{g} / \mathrm{ml})$ was coated on 96 -well microtiter plates at $4^{\circ} \mathrm{C}$ overnight. Mouse serum samples were added to the wells and incubated at room temperature for $1 \mathrm{~h}$ with gentle shaking. Then each well was incubated with $100 \mu \mathrm{l}$ of HRP conjugated anti-mouse IgG diluted in 1:250, anti-mouse IgG1 or IgG2a (1:500) for $1 \mathrm{~h}$. After added substrate solution ( $\mathrm{pH} 4.0)(1.05 \%$ citrate substrate buffer; $1.5 \%$ ABTS; $0.03 \% \mathrm{H}_{2} \mathrm{O}_{2}$ ) for $20 \mathrm{~min}$, the absorbance was measured at $405 \mathrm{~nm}$. All measurements were performed in triplicate.

\section{Lymphocyte proliferation assay by MTS}

Two weeks after the last immunization, three mice per group were euthanized, and their splenocytes were aseptically harvested through a wire mesh and then purified by removing the red blood cells using RBC lysis solution (Sigma, USA). The lymphocytes from each group were then cultured in triplicate at a density of $2 \times 10^{5}$ cells per well in complete medium (DMEM medium $+10 \%$ FCS $+100 \mathrm{U} / \mathrm{ml}$ penicillin/streptomycin). The cells were stimulated with TLA $(10 \mu \mathrm{g} / \mathrm{ml})$, concanavalin A (ConA; $5 \mu \mathrm{g} / \mathrm{ml}$; Sigma) or medium alone served as positive and negative controls respectively, at $37^{\circ} \mathrm{C}$ in a $5 \% \mathrm{CO}_{2}$ incubator. The proliferative activity was measured using MTS method (Promega, USA) after four days. The stimulation index (SI) was calculated by using the formula $\left(\mathrm{OD}_{570 \mathrm{TLA}} /\right.$ $\left.\mathrm{OD}_{570 \mathrm{M}}\right)$ : $\left(\mathrm{OD}_{570 \text { ConA }} / \mathrm{OD}_{570 \mathrm{M}}\right)$.

\section{Flow cytometry}

The $\mathrm{T}$ cell subclasses, CD4+ and CD8+ in spleen were determined to the percentage using flow cytometry with staining by phycoerythrin (PE)-labeled anti-mouse CD3 (eBioscience), Allophycocyanin (APC)-labeled antimouse CD4 (eBioscience) and fluorescein isothiocyanate (FITC)-labeled anti-mouse CD8 (eBioscience) antibodies. After washing by PBS, the cells were fixed with FACScan buffer (PBS containing 1\% BSA and 0.1\% Sodium azide) and\% paraformaldehyde. All the samples were analyzed of fluorescence profiles on a FACScan flow cytometer (BD Biosciences) using SYSTEM II software (Coulter).

\section{Cytokine assays}

The splenocytes without RBC from each group were cocultured with TLA for positive control and medium alone for negative control in 96-well flat-bottom microtiter plates as described in the section of lymphocyte proliferation assay. Culture supernatants were harvested at $24 \mathrm{~h}$ for determination of IL-2 and IL-4, $72 \mathrm{~h}$ for IL-10, and $96 \mathrm{~h}$ for IL-23, IFN- $\gamma$ and IL-12(p70) using commercial ELISA kits according to the manufacturer's instructions (Biolegend, USA). The analysis was performed with the data from three independent experiments.

\section{Statistical analysis}

All statistical analyses were performed following the procedure of SAS (Statistical Analysis System, Version 8.0). The differences of each variable including antibody responses, lymphoproliferation assays, cytokine production, and percentages of $\mathrm{CD} 4+$ and $\mathrm{CD} 8+\mathrm{T}$ cells among all the groups were compared by one-way ANOVA. The level of significant difference in comparisons between groups was defined as $P<0.05$. 


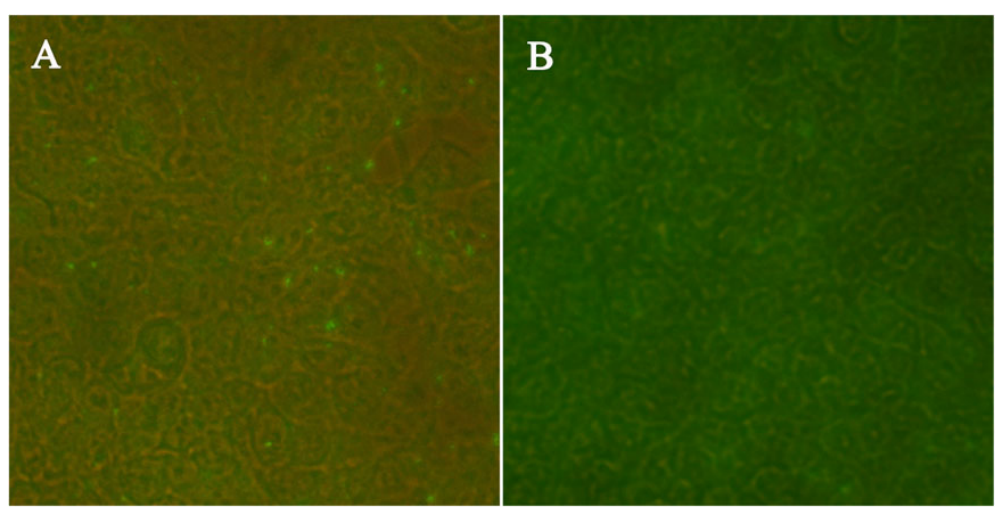

Figure 1 Analysis of TgCDPK3 expression in HEK293 cells by indirect immunofluorescence (IFA) at 48 h post-transfection. (A) HEK293 cells were transfected with pVAX-CDPK3; (B) empty vector pVAX I.

\section{Results}

Detection of the eukaryotic and prokaryotic expression of TgCDPK3

The expression of pVAX-CDPK3 was identified using IFA. As shown in Figure 1, specific green fluorescence was observed in HEK293 cells transfected with the eukaryotic recombinant plasmid pVAX-CDPK3, but not in the negative controls that transfected with the same amount of empty pVAX I. These results revealed that the $\operatorname{TgCDPK} 3$ protein was expressed successfully in HEK293 cells.

E. coli BL21 (DE3) transferred with pET-CDPK3 was separated by SDS-PAGE. After staining by Coomassie brilliant blue, the $\mathrm{rTgCDPK} 3$ protein was shown at the position of approximately $70 \mathrm{kDa}$, which was consistent with the theoretical value (Figure 2).

\section{Western blotting and antibody responses}

To determine the reactogenicity of $\mathrm{rTgCDPK} 3$ protein, western blotting showed that the anti-CDPK3 antibody could recognize the protein at the position of approximately $70 \mathrm{kDa}$. No band was found when the protein reacted with the negative sera (Figure 2).

The levels of specific antibody response were measured by ELISA. As shown in Figure 3A, a higher level of specific IgG antibodies was detected in those mice immunized with pVAX-CDPK3 $(P<0.05)$, and the OD values in pVAX-CDPK3 group reached to a significantly high level with successive immunization $(P<0.05)$. In contrast, antibodies in mice from three control groups did not statistically increase with injection. In order to characterize whether a Th1 and/or Th2 response was elicited in immunized mice, the subclasses of IgG (IgG1 and IgG2a) specific to $\mathrm{rTgCDPK} 3$ were analyzed individually in sera from all groups at two weeks after the last immunization. A mixed IgG1/IgG2a response was determined in the sera of mice immunized with pVAX-CDPK3 (Figure 3B), and OD values of IgG2a was observed statistically higher than $\operatorname{IgG1}(P<0.05)$. All these results indicated that both a specific humoral response and the Th1 type immune response were elicited.

\section{Evaluation of splenocyte proliferation}

The proliferative response was observed after $96 \mathrm{~h}$ of splenocytes co-cultured with stimulant. As shown in Table 1, the proliferation SI from pVAX-CDPK3 vaccinated group $(1.30 \pm 0.18)$ was significantly increased when compared with vaccination with PBS $(0.91 \pm 0.01)$, pVAX I $(0.89 \pm 0.07)$ or blank control $(0.97 \pm 0.01)$.

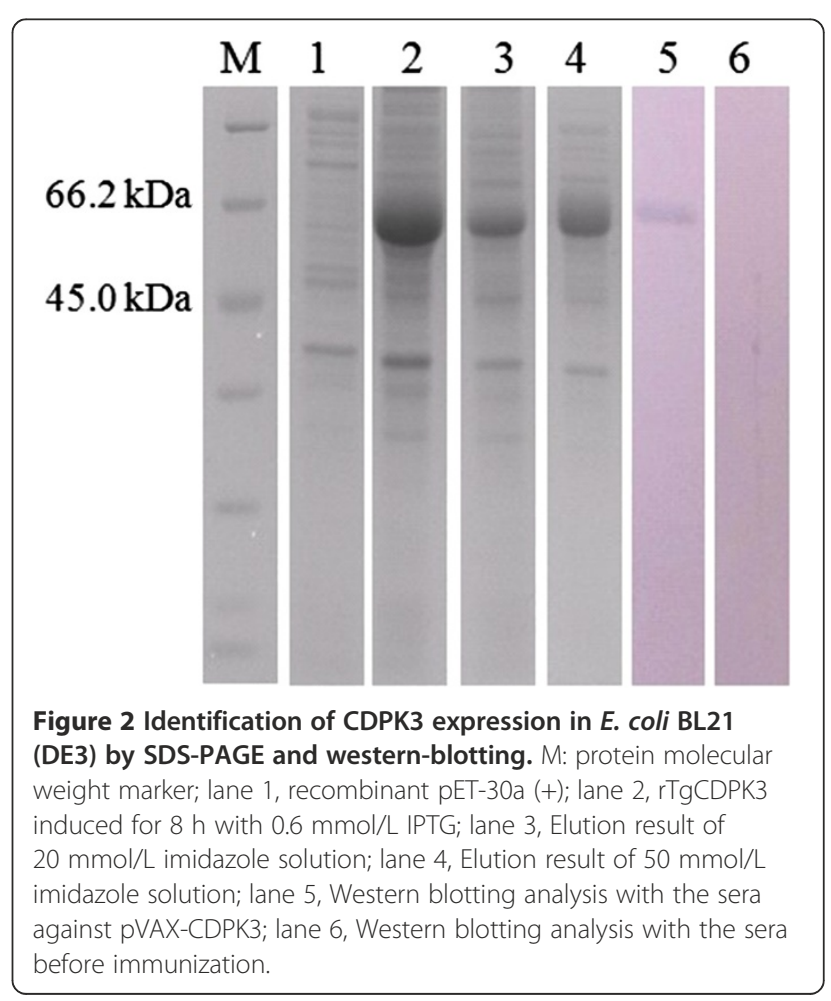




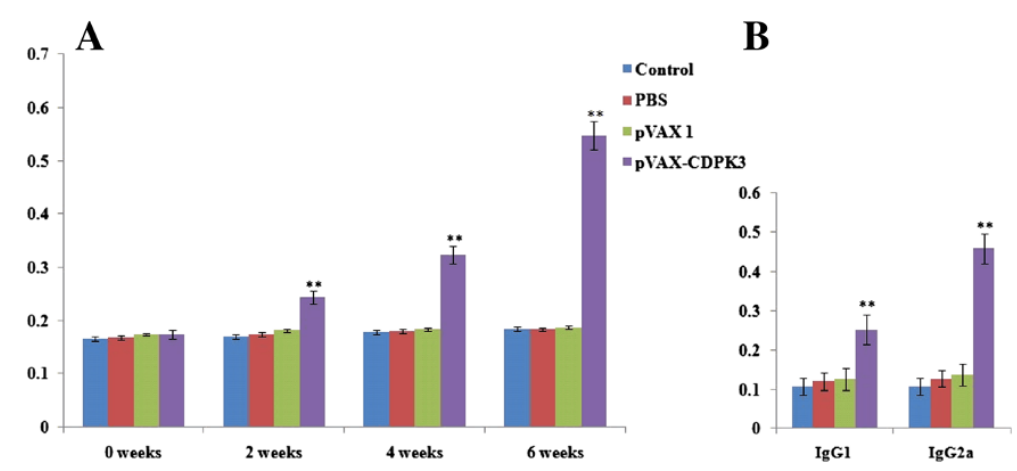

Figure 3 Specific antibody response induced by DNA immunization with pVAX-CDPK3, pVAX I, PBS and blank controls using ELISA. (A) Determination of specific anti-TgCDPK3 IgG antibodies in the sera of Kunming mice at 0, 2, 4, and 6 weeks. (B) Determination of the specific anti-TgCDPK3 IgG subclass profile (IgG1 or lgG2a) in the sera of Kunming mice two weeks after the last immunization. Each bar represents the mean OD $( \pm$ S.E., $n=3)$. Statistically significant differences of OD values $(P<0.05)$ are indicated by $(* *)$.

\section{Percentages of CD4+ and CD8+ T lymphocyte}

As shown in Table 1, the percentages of CD3+ CD4+ T lymphocytes in pVAX-CDPK3 group (CD4+ with $20.33 \pm$ 3.06) were higher than those in PBS group (CD4+ with $13.70 \pm 2.41$ ), in pVAX I group (CD4+ with $14.17 \pm 1.88)$, or in blank control (CD4+ with $15.27 \pm 1.89)(P<0.05)$. The ratio of $\mathrm{CD} 3+\mathrm{CD} 8+\mathrm{T}$ cells in the spleen of vaccinated mice was slightly higher than that in all the controls, but the difference was not statistically significant $(P>$ $0.05)$. There was no difference in terms of the percentages of CD3+ CD4+ and CD3+ CD8+ T lymphocytes among the three control groups.

\section{Cytokine production by spleen cells}

Two weeks after the final immunization, spleen cell suspensions from individual mice were stimulated in vitro with TLA and were qualified with ELISA. As shown in Table 2, a significantly high level of IFN- $\gamma$, IL-23, IL-12 (p70) and IL-2 was observed in spleen cell cultures from mice immunized with pVAX-CDPK3 compared with that in the pVAX I, PBS and the blank control groups $(P<0.05)$. In the meanwhile, the levels of IL- 4 and IL-10 were also slightly increased in supernatants from spleen

Table 1 Splenocyte proliferative responses and the percentages of $T$ cell subsets in immunized mice 2 weeks after the last immunization

\begin{tabular}{llll}
\hline Groups & SI (Mean \pm SD) & $\begin{array}{l}\text { CD3 + CD4 } \\
\text { CD8 }-(\%)\end{array}$ & $\begin{array}{l}\text { CD3 + CD8 + } \\
\text { CD4- (\%) }\end{array}$ \\
\hline pVAX-CDPK3 & $1.30 \pm 0.18^{* *}$ & $20.33 \pm 3.06^{* *}$ & $8.90 \pm 2.95$ \\
pVAXI & $0.89 \pm 0.07$ & $14.17 \pm 1.88$ & $6.47 \pm 1.20$ \\
PBS & $0.91 \pm 0.01$ & $13.70 \pm 2.41$ & $6.37 \pm 1.25$ \\
Blank control & $0.97 \pm 0.01$ & $15.27 \pm 1.89$ & $5.03 \pm 0.40$ \\
\hline
\end{tabular}

SI stands for stimulation index.

**Statistically significant difference $(P<0.05)$. cells of mice immunized with pVAX-CDPK3 compared to these control groups $(P<0.05)$.

Protective activity induced by vaccination with pVAX-CDPK3

To determine whether pVAX-CDPK3 can induce effective protection against $T$. gondii acute infection, 10 mice from each group were intraperitoneally challenged with a lethal dose of tachyzoites of the virulent $T$. gondii $\mathrm{RH}$ strain two weeks after the last immunization. The average survival time of immunized mice was significantly longer than that of the control groups (Figure 4). Mice injected with pVAX I, PBS and blank control did not reveal significant differences in their survival time $(P>0.05)$.

To evaluate whether immune responses generated against pVAX-CDPK3 were sufficient for providing protection against the formation of $T$. gondii tissue cysts in the brain, all the groups of mice were challenged with 10 cysts of $T$. gondii PRU strain per mouse and the brain cysts loads were assessed 30 days after challenge. As shown in Table 3, mice immunized with pVAX-CDPK3 produced significantly fewer brain cysts than the control groups $(P<0.05)$.

\section{Discussion}

T. gondii can infect all types of nucleated cells and evolves several pathways to assure entry-exit from the cells [17]. Plant-like CDPKs are considered to play important signaling roles in the signal transduction cascades. Among them, TgCDPK 3 activity, which is likely determined by calcium and potassium concentration, can control the calcium-dependent permeabilization of the parasitophorous vacuole membrane (PVM) and regulate microneme secretion and thus plays a vital role in the rapid induction of parasite egress $[15,18]$. Considering the crucial biological characteristics of TgCDPK3, we 
Table 2 Cytokine production by splenocytes of immunized Kunming mice after stimulation by toxoplasma lysate antigen $^{\mathrm{a}}$

\begin{tabular}{lllllll}
\hline Groups & \multicolumn{4}{l}{ Cytokine production $\mathbf{( p g} / \mathbf{m l})$} & & \\
\cline { 2 - 7 } & IFN- $\boldsymbol{l}$ & IL-2 & IL-4 & IL-10 & IL-12 (p70) & IL-23 \\
\hline pVAX-CDPK3 & $612.11 \pm 128.56^{* *}$ & $263.79 \pm 35.43^{* *}$ & $32.42 \pm 2.74^{* *}$ & $870.12 \pm 226.50^{* *}$ & $129.63 \pm 77.42^{* *}$ & $87.59 \pm 7.14^{* *}$ \\
pVAXI & $34.91 \pm 11.23$ & $<15$ & $<15$ & $322.50 \pm 179.17$ & $15.14 \pm 7.63$ & $19.93 \pm 22.65$ \\
PBS & $36.34 \pm 4.83$ & $<15$ & 18.96 & $356.13 \pm 175.27$ & $22.31 \pm 4.40$ & $27.12 \pm 18.46$ \\
Blank control & $47.28 \pm 16.60$ & $<15$ & $<15$ & $477.25 \pm 87.53$ & $22.65 \pm 21.90$ & $23.68 \pm 14.13$ \\
\hline
\end{tabular}

${ }^{\mathrm{a}}$ Splenocytes from mice were harvested two weeks after the last immunization.

**Statistically significant difference $(P<0.05)$.

firstly studied the various immune responses elicited by immunization with the DNA vaccine coding TgCDPK3 protein and evaluated its vaccinal potentiality.

In recent years, many studies have evaluated the protective immune responses elicited by different single antigens including ADF, NTPase, IMP, ROM1, ROP18 and elF4A [16,19-23], which showed partial protection against $T$. gondii in mice model. In the present study, vaccination of Kunming mice with pVAX-CDPK3 can induce high levels of specific humoral and cellular immune responses, resulting in effective protective immunity, showing increased survival time (RH strain: $13.5 \pm$ 4.89 days, $P<0.05$ ) and reduced brain cysts (PRU strain: $50 \%, P<0.05)$ contrasted with the control mice, which demonstrated that TgCDPK3 is a promising DNA vaccine candidate against toxoplasmosis.

Specific antibody response has been considered to be important in immunity against $T$. gondii. In the present study, the mice immunized intramuscularly with pVAX-

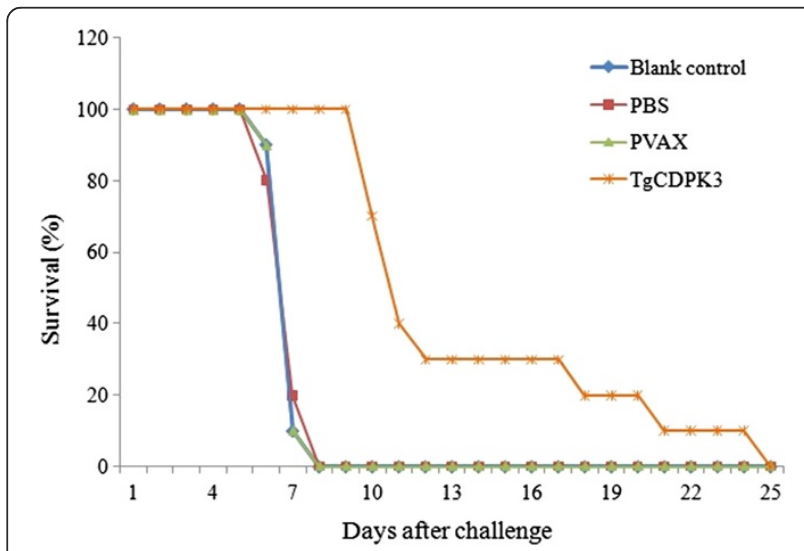

Figure 4 Survival rate of mice immunized with pVAX-CDPK3, pVAX I, PBS and nothing followed by challenge with $1 \times 10^{3}$ tachyzoites 2 weeks after the last immunization. The mice immunized with pVAX-CDPK3 were dead from day 10 to 24 that showed an increased survival time (13.5 \pm 4.89 days) compared with mice in the control groups ( $\mathrm{PVAX} 1, \mathrm{PBS}$, blanking controls) died within 7 days after challenge $(P<0.05)$.
CDPK3 produced specific antibodies against $T$. gondii CDPK3, which may have the ability to kill the parasite by the attachment of the parasite to the host cell receptors, or the complement protein [24,25].

Due to the obligate intracellular lifestyle, $\mathrm{T}$ cellmediated adaptive immune responses involving in CD4+ and CD8+ T cells are known to be important in resistance against primary $T$. gondii infection and reactivation of chronic toxoplasmosis [26-28]. In the present study, a significantly proliferative response of splenocytes from pVAX-CDPK3 vaccinated mice indicated that vigorous cellular immune responses were induced by the DNA vaccine. Furthermore, similar to the results from DNA vaccination with ROP2, actin depolymerizing factor protein (ADP) and rhomboid protein (ROM1) of $T$. gondii [29-31], the percentages of both CD4+ T cells were also increased in pVAX-CDPK3 immunized mice, which again stated an activated CD4+ T cells immune response.

It is well established that the development of Th1-type lymphocytes are considered to play a critical role in the protective immunity against $T$. gondii [32-34]. So, the present study further analyzed the CD4+ T cells with a helper $\mathrm{T}$ cell type 1 (Th1) cytokine secretion profile and antibodies subclass. Under our experimental conditions, a mixed humoral response (IgG1/ IgG2a) with high ratio of IgG2a to IgG1 antibody titers was observed in the pVAX-CDPK3 vaccinated mice, and Th1 type response correlative cytokines (IFN- $\gamma$, IL-2 and IL-12) released from spleen cells of vaccinated mice were observed. The low level increase of Th2 cytokines (IL-4 and IL-10)

Table 3 Mean tissue cyst load in the brain of immunized mice after challenge with 10 cysts of $T$. gondii PRU strain

\begin{tabular}{ll}
\hline Groups & Number of brain cysts (Means \pm SD) \\
\hline pVAX I & $3377.78 \pm 226.08$ \\
PBS & $3166.67 \pm 258.77$ \\
Blank control & $3232.78 \pm 196.64$ \\
pVAX-CDPK3 & $1566.67 \pm 167.10^{* *}$ \\
\hline
\end{tabular}

**Statistically significant difference $(P<0.05)$. 
induced by pVAX-CDPK3, which is also detected in the mice immunized with eIF4A, MIC6 and PLP $[19,35,36]$, is likely because the antigen in the cytosol of antigen presenting cells (APCs) was not only processed and presented by MHC Class I molecules but was also present by MHC Class II molecules. These finding revealed that a predominant Th1 type cell immune response has been driven by immunization with pVAX-CDPK3.

Cytokines play an important role in immunity against T. gondii infection by both stimulating macrophages and CTL protective immunity during the innate and adaptive immune response $[37,38]$. IL- 4 and IL-10 can promote the proliferation and differentiation of activated B cells, mast cells and peripheral lymphocytes and were showed to play an important role during the early phase of acute T. gondii infection $[39,40]$, which partially contributed to protection against the acute stage of $T$. gondii infection in the present study. IL-23 secreted by activated macrophages and dendritic cells has been shown to enhance the production of the most critical cytokine, IFN- $\gamma$, in mediating host protection against $T$. gondii infection. IFN- $\gamma$, which was produced by T cells and NK cells, can restrict the growth of $T$. gondii in the acute phase of the infection and the reactivation of parasites from dormant cysts through triggering lysosomal activity, inducing nitric oxide production and modulating metabolic activity of some antigen presenting cells [38,41,42]. Furthermore, IFN- $\gamma$ in combined with IL- 2 and IL-12 is involved in the protection against parasitic invasions [37]. Therefore, our results of the high level of IFN- $\gamma$, IL-2, IL-12 (p70) and IL-23 in the mice immunized with pVAX-CDPK3 suggested that these Th1-type cytokines confer host resistance against $T$. gondii infection.

\section{Conclusions}

The present study evaluated, for the first time, the immunogenicity and protective potency of a DNA vaccine encoding $\mathrm{TgCDPK} 3$, and demonstrated that $\mathrm{TgCDPK} 3$ is a potential vaccine candidate against acute and chronic toxoplasmosis in the mice model, and warrant further studies in other apicomplexan parasites.

\section{Competing interests}

The authors declare that they have no competing interests.

\section{Authors' contributions}

XQZ, NZZ and JC conceived and designed the study, and critically revised the manuscript. NZZ, DHZ, WPT, YX and JL performed the experiments. NZZ, $\mathrm{JC}$ and SYH analyzed the data and drafted the manuscript. All authors read and approved the final manuscript.

\section{Acknowledgements}

Project support was provided by the National Natural Science Foundation of China (Grant Nos. 31101812, 31230073 and 31172316), and the Science Fund for Creative Research Groups of Gansu Province (Grant No. 1210RJIA006).

\section{Author details}

'State Key Laboratory of Veterinary Etiological Biology, Key Laboratory of Veterinary Parasitology of Gansu Province, Lanzhou Veterinary Research Institute, Chinese Academy of Agricultural Sciences, Lanzhou, Gansu Province 730046, PR China. ${ }^{2}$ College of Animal Science and Technology, Anhui Agricultural University, Hefei, Anhui Province 230036, PR China. ${ }^{3}$ College of Veterinary Medicine, Northeast Agricultural University, Harbin, Heilongjiang Province 150030, PR China. ${ }^{4}$ College of Veterinary Medicine, South China Agricultural University, Guangzhou, Guangdong Province 510642, PR China. ${ }^{5}$ College of Animal Science and Veterinary Medicine, Heilongjiang Bayi Agricultural University, Daqing, Heilongjiang Province 163319, PR China.

Received: 5 August 2013 Accepted: 29 October 2013

Published: 31 October 2013

\section{References}

1. Dubey JP: Toxoplasmosis of animals and humans. Second editionth edition. Boca Raton, Florida: CRC Press; 2010:1-313.

2. Robert-Gangneux F, Darde ML: Epidemiology of and diagnostic strategies for toxoplasmosis. Clin Microbiol Rev 2012, 25:264-296.

3. Duan G, Tian YM, Li BF, Yang JF, Liu ZL, Yuan FZ, Zhu XQ, Zou FC: Seroprevalence of Toxoplasma gondii infection in pet dogs in Kunming Southwest China. Parasit Vectors 2012, 5:118.

4. Gebremedhin EZ, Abebe AH, Tessema TS, Tullu KD, Medhin G, Vitale M, Di Marco V, Cox E, Dorny P: Seroepidemiology of Toxoplasma gondii infection in women of child-bearing age in central Ethiopia. BMC Infect Dis 2013, 13:101.

5. Elmore SA, Jones JL, Conrad PA, Patton S, Lindsayand DS, Dubey JP: Toxoplasma gondii: pidemiology, feline clinical aspects, and prevention. Trends Parasitol 2010, 26:190-196.

6. Zhou P, Chen ZG, Li HL, Zheng HH, He SY, Lin RQ, Zhu XQ: Toxoplasma gondii infection in humans in China. Parasit Vectors 2011, 4:165.

7. Dubey JP, Lago EG, Gennari SM, Su C, Jones JL: Toxoplasmosis in humans and animals in Brazil: high prevalence, high burden of disease, and epidemiology. Parasitology 2012, 139:1375-1424.

8. Samra NA, McCrindle CM, Penzhorn BL, Cenci-Goga BT: Seroprevalence of toxoplasmosis in sheep in South Africa. J S Afr Vet Assoc 2007, 78:116-120.

9. Innes EA, Vermeulen AN: Vaccination as a control strategy against the coccidial parasites Eimeria, Toxoplasma and Neospora. Parasitology 2006, 133:145-168.

10. Innes EA: Vaccination against Toxoplasma gondii: an increasing priority for collaborative research? Expert Rev Vaccines 2010, 9:1117-1119.

11. Buxton $D$, Innes EA: A commercial vaccine for ovine toxoplasmosis. Parasitology 1995, 110:11-16.

12. Jongert E, Roberts CW, Gargano N, Forster-Waldl E, Petersen E: Vaccines against Toxoplasma gondii: challenges and opportunities. Mem Inst Oswaldo Cruz 2009, 104:252-266.

13. Zhao G, Zhou A, Lv G, Meng M, Sun M, Bai Y, Han Y, Wang L, Zhou H, Cong H, Zhao Q, Zhu XQ, He S: Toxoplasma gondii cathepsin proteases are undeveloped prominent vaccine antigens against toxoplasmosis. BMC Infect Dis 2013, 13:207.

14. Moreno SN, Docampo R: Calcium regulation in protozoan parasites. Curr Opin Microbiol 2003, 6:359-364.

15. McCoy JM, Whitehead L, van Dooren GG, Tonkin CJ: TgCDPK3 regulates calcium-dependent egress of Toxoplasma gondii from host cells. PLoS Pathog 2012, 8:1003066.

16. Yuan ZG, Zhang XX, Lin RQ, Petersen E, He S, Yu M, He XH, Zhou DH, He Y, Li HX, Liao M, Zhu XQ: Protective effect against toxoplasmosis in mice induced by DNA immunization with gene encoding Toxoplasma gondii ROP18. Vaccine 2011, 29:6614-6619.

17. Sibley LD: Invasion and intracellular survival by protozoan parasites. Immunol Rev 2011, 240:72-91.

18. Garrison E, Treeck M, Ehret E, Butz H, Garbuz T, Oswald BP, Settles M, Boothroyd J, Arrizabalaga G: A forward genetic screen reveals that calcium-dependent protein kinase 3 regulates egress in Toxoplasma. PLoS Pathog 2012, 8:1003049.

19. Chen J, Huang SY, Li ZY, Yuan ZG, Zhou D, Petersen E, Zhang NZ, Zhu XQ: Protective immunity induced by a DNA vaccine expressing elF4A of Toxoplasma gondii against acute toxoplasmosis in mice. Vaccine 2013, 31:1734-1739. 
20. Zhang NZ, Chen J, Wang M, Petersen E, Zhu XQ: Vaccines against Toxoplasma gondii: new developments and perspectives. Expert Rev Vaccines 2013, 12:1287-1299.

21. Li J, Huang $X$, Zhang G, Gong P, Zhang X, Wu L: Immune response and protective efficacy against homologous challenge in BALB/c mice vaccinated with DNA vaccine encoding Toxoplasma gondii actin depolymerizing factor gene. Vet Parasitol 2011, 179:1-6.

22. Tan F, Hu X, Luo FJ, Pan CW, Chen XG: Induction of protective Th1 immune responses in mice by vaccination with recombinant Toxoplasma gondii nucleoside triphosphate hydrolase-II. Vaccine 2011, 29:2742-2748.

23. Cui X, Lei T, Yang D, Hao P, Li B, Liu Q: Toxoplasma gondii immune mapped protein-1 (TgIMP1) is a novel vaccine candidate against toxoplasmosis. Vaccine 2012, 30:2282-2287.

24. Kang H, Remington JS, Suzuki Y: Decreased resistance of B cell deficient mice to infection with Toxoplasma gondii despite unimpaired expression of IFN-gamma, TNF-alpha and inducible nitric oxide synthase. J Immunol 2000, 164:2629-2634.

25. Sibley LD, Adams LB, Krahenbuhl JL: Macrophage interactions in toxoplasmosis. Res Immunol 1993, 144:38-40.

26. Brown CR, McLeod R: Class I MHC genes and CD8+ T cells determine cyst number in Toxoplasma gondii infection. J Immunol 1990, 145:3438-3441.

27. Gazzinelli $R, X u$ Y, Hieny S, Cheever A, Sher A: Simultaneous depletion of $\mathrm{CD} 4+$ and $\mathrm{CD} 8+\mathrm{T}$ lymphocytes is required to reactivate chronic infection with Toxoplasma gondii. J Immunol 1992, 149:175-180.

28. Jordan KA, Hunter CA: Regulation of CD8+ T cell responses to infection with parasitic protozoa. Exp Parasitol 2010, 126:318-325.

29. Li J, Han Q, Gong P, Yang T, Ren B, Li S, Zhang X: Toxoplasma gondii rhomboid protein 1 (TgROM1) is a potential vaccine candidate against toxoplasmosis. Vet Parasitol 2012, 184:154-160.

30. Huang X, Li J, Zhang G, Gong P, Yang J, Zhang X: Toxoplasma gondii: protective immunity against toxoplasmosis with recombinant actin depolymerizing factor protein in balb/c mice. Exp Parasito/ 2012, 130:218-222.

31. Wang H, Liu Q, Liu K, Zhong W, Gao S, Jiang L, An N: Immune response induced by recombinant Mycobacterium bovis BCG expressing ROP2 gene of Toxoplasma gondii. Parasitol Int 2007, 56:263-268.

32. Gazzinelli RT, Wysocka M, Hayashi S, Denkers EY, Hieny S, Caspar P, Trinchier G, Sher A: Parasite-induced IL-12 stimulates early IFN- synthesis and resistance during acute infection with Toxoplasma gondii. J Immunol 1994, 153:2533-2543.

33. Hunter CA, Subauste CS, Van Cleave VH, Remington JS: Production of gamma interferon by natural killer cells from Toxoplasma gondii-infected SCID mice: regulation by interleukin-10, interleukin-12, and tumor necrosis factor alpha. Infect Immun 1994, 62:2818-2824.

34. Gazzinelli R, Hakim FT, Hieny S, Shearer GM, Sher A: Synergistic role of CD4 + and CD8+ T lymphocytes in IFN-production and protective immunity induced by an attenuated Toxoplasma gondii vaccine. J Immunol 1991 146:286-292.

35. Yan HK, Yuan ZG, Song HQ, Petersen E, Zhou Y, Ren D, Zhou DH, Li HX, Lin $R Q$, Yang $G L$, Zhu $X Q$ : Vaccination with a DNA vaccine coding for perforin-like protein 1 and MIC6 induces significant protective immunity against Toxoplasma gondii. Clin Vaccine Immunol 2012, 19:684-689.

36. Peng GH, Yuan ZG, Zhou DH, He XH, Liu MM, Yan C, Yin CC, He Y, Lin RQ, Zhu XQ: Toxoplasma gondii microneme protein 6 (MIC6) is a potential vaccine candidate against toxoplasmosis in mice. Vaccine 2009, 27:6570-6574.

37. Matowicka-Karna J, Dymicka-Piekarska V, Kemona H: Does Toxoplasma gondii infection affect the levels of IgE and cytokines (IL-5, IL-6, IL-10, IL12, and TNF-alpha)? Clin Dev Immunol 2009, 2009:374696.

38. Sturge CR, Benson A, Raetz M, Wilhelm CL, Mirpuri J, Vitetta ES, Yarovinsky F: TLR-independent neutrophil-derived IFN- $\gamma$ is important for host resistance to intracellular pathogens. Proc Natl Acad Sci USA 2013, 110:10711-10716.

39. Bessieres MH, Swierczynski B, Cassaing S, Miedouge M, Olle P, Sequela JP, Pipy B: Role of IFN-gamma, TNF-alpha, IL4 and IL10 in the regulation of experimental Toxoplasma gondii infection. J Eukaryot Microbiol 1997, 44:87S.

40. Gazzinelli RT, Wysocka M, Hieny S, Scharton-Kersten T, Cheever A, Kuhn R, Muller W, Trinchieri G, Sher A: In the absence of endogenous IL-10, mice acutely infected with Toxoplasma gondii succumb to a lethal immune response dependent on CD4 + T cells and accompanied by overproduction of IL-12, IFN-gamma and TNF-alpha. J Immunol 1996, 157:798-805.

41. MacMicking JD: Interferon-inducible effector mechanisms in cell-autonomous immunity. Nat Rev Immunol 2012, 12:367-382.

42. Taylor GA, Feng CG, Sher A: p47 GTPases: regulators of immunity to intracellular pathogens. Nat Rev Immunol 2004, 4:100-109.

doi:10.1186/1471-2334-13-512

Cite this article as: Zhang et al:: Protective immunity against Toxoplasma gondii induced by DNA immunization with the gene encoding a novel vaccine candidate: calcium-dependent protein kinase 3. BMC Infectious Diseases 2013 13:512.

\section{Submit your next manuscript to BioMed Central and take full advantage of:}

- Convenient online submission

- Thorough peer review

- No space constraints or color figure charges

- Immediate publication on acceptance

- Inclusion in PubMed, CAS, Scopus and Google Scholar

- Research which is freely available for redistribution

Submit your manuscript at www.biomedcentral.com/submit
C) Biomed Central 